Treatment based on molecular profiling of tumor is advertised however there are very limited clinical data supporting this approach so far. Only one, relatively small, randomized clinical trial (SHIVA) have not met its primary endpoint - prolongation of PFS. Some other unpublished series were reported during ASCO 2017 and are discussed in this review. There are many issues to be resolved before the tumor profiling will enter the clinical practice with significant benefit for patients, eg. spatial and temporal heterogeneity of tumor cells in individual patient, wide access to targeted therapies, toxicity of combined targeted therapies.

Key words: tumor profiling, targeted therapies.

Contemp Oncol (Pozn) 2018; 22 (1A): 3-6 DOI: https://doi.org/10.5114/wo.2018.73873

\section{Molecular profiling of tumours for precision oncology - high hopes versus reality}

\author{
Sergiusz Nawrocki
}

Oncology and Radiotherapy Chair, Medical University of Silesia, Katowice, Poland

Implementation of imatinib, trastuzumab and vemurafenib to clinical practice has opened a new era of genomic-driven medicine in oncology. Imatinib in chronic myeloid leukaemia (CML) prolongs overall survival (OS) of the treated patients to the level of OS reached by the general population. Trastuzumab in HER2-expressing breast cancer allows for a 35-50\% reduction of mortality in the adjuvant setting and a $20 \%$ reduction of mortality of metastatic patients. Vemurafenib in BRAF-mutated melanoma patients extends survival but unfortunately most patients have progression after several months of response. These 3 drugs were taken as exemplary milestones in precision oncology because they have paved new roads but also because they have shown us some important limitations of targeted drugs. Imatinib is extremely effective in CML where the target - bcr-abl kinase - is a real driver oncogene and CML cells are oncogene-addicted; if bcr-abl kinase is switched off by imatinib, the CML cells are not able to survive. However, in contrast to $\mathrm{CML}$, solid tumours with the same genetic aberration are much less sensitive to imatinib because other known and unknown genetic alterations are driving their growth together or independently of bcr-abl kinase. Trastuzumab is highly effective in HER2-expressing breast cancer, moderately affective (lower response rates and response duration in patients) in HER2-expressing gastric cancer patients and marginally or not effective in different HER2-expressing solid tumours. Vemurafenib has demonstrated high activity in BRAF-mutated melanoma patients but fast and inevitable progression of disease is observed in all responders after a relatively short period of time.

Precision oncology assumes that individual comprehensive molecular profiling of the patient's tumour sample is able to define a draggable target or even multiple targets which enable one to tailor treatment better than that based on current clinical experience and the histopathology report. In the US but also in other countries, including Poland, molecular profiling is marketed directly to patients despite lack or very vague evidence of benefit of such an approach so far. Only one randomised clinical trial (SHIVA) has been published and a few large clinical series of patients have been evaluated. Table 1 shows clinical studies that have evaluated molecular profiling of patients.

Updated results of studies highlighted above and some other series were reported at the ASCO Annual Conference 2017 which was held in Chicago at the beginning of June.

Olivier Tredan and coworkers presented results of the ProfiLER Study at ASCO 2017. ProfiLER (NCT01774409) is a molecular profiling clinical trial exploring cancer cell genomic alterations in patients with advanced disease to guide treatment. Patients with confirmed diagnosis of advanced cancers are eligible for ProfiLER. DNA is extracted from either archival or fresh collected tumour samples and is analyzed by targeted exon sequencing of 60 cancer related genes and whole genome array comparative genomic hybridization (CGH). A multidisciplinary molecular board analyses genomic data 
Table 1. Clinical studies that have evaluated molecular profiling of patients

\begin{tabular}{|c|c|c|c|c|c|c|}
\hline Study & Design & $\begin{array}{l}\text { Screened } \\
\text { sample }\end{array}$ & $\begin{array}{l}\text { Patients } \\
\text { with genetic } \\
\text { profile }\end{array}$ & $\begin{array}{l}\text { Patients with } \\
\text { draggable } \\
\text { mutation }\end{array}$ & $\begin{array}{l}\text { Patients } \\
\text { received } \\
\text { matched drug }\end{array}$ & Outcome \\
\hline SHIVA3 & Randomised trial & 741 patients & $67 \%$ & $40 \%$ & 96 & $\begin{array}{l}\text { No significant difference in } \\
\text { PFS (primary endpoint) }\end{array}$ \\
\hline $\begin{array}{l}\text { Lung Cancer Mutation } \\
\text { Consortium (Study I and II) }\end{array}$ & $\begin{array}{l}\text { Testing for driver } \\
\text { mutations }\end{array}$ & & & & & $\begin{array}{l}\text { Longer OS in mutation } \\
\text { treated with directed } \\
\text { therapy }\end{array}$ \\
\hline Study I1 & & 1007 patients & $73 \%$ & $46 \%$ & 260 & \\
\hline Study 112 & & 1315 patients & $70 \%$ & $5.6 \%$ & 127 & \\
\hline SAFIR4 & $\begin{array}{l}\text { Treatment chosen } \\
\text { after genetic } \\
\text { profiling }\end{array}$ & $\begin{array}{l}423 \text { patients } \\
\text { with breast } \\
\text { cancer }\end{array}$ & $71 \%$ & $46 \%$ & 55 & $\begin{array}{c}3 \% \text { of screened sample } \\
\text { partial response or stable } \\
\text { disease }\end{array}$ \\
\hline M.D. Anderson Study5 & $\begin{array}{l}\text { Treatment chosen } \\
\text { after gene seq. }\end{array}$ & 2601 patients & $77 \%$ & $30 \%$ & 83 & \\
\hline IMPACT-COMPACT6 & $\begin{array}{l}\text { Treatment chosen } \\
\text { after gene seq. } \\
\text { from archival } \\
\text { tissue }\end{array}$ & 1893 patients & $87 \%$ & $10 \%$ & 84 & $\begin{array}{c}\text { Response rate } 20 \% \text { vs. } 11 \% \\
\text { in unmatched patients }\end{array}$ \\
\hline Cleveland Clinic Study7 & $\begin{array}{l}\text { Treatment chosen } \\
\text { after gene seq. }\end{array}$ & 250 patients & $89 \%$ & $44 \%$ & 24 & \\
\hline
\end{tabular}

and recommends molecular targeted therapies (MTT) when actionable alterations are found. As of January 2017, 2490 patients (55\% female, median age 59, range 1-90) had consented; 1826 (73.3\%) tumours were analysed, 301 (12\%) are ongoing (not done in 363 patients (14.6\%) due to technical issues). Tumour types were colorectal (10.3\%), gynaecological (9.5\%), breast (8.8\%), head and neck (7.1\%) carcinomas, sarcomas (7.1\%) and brain tumours (6.5\%). 940/1826 patients (51.5\%) had at least 1 actionable mutation (AM): 579 patients with only one AM, while 358 with 2 or more AM (up to 6). Mutations (including substitutions and small indels), amplifications and homozygous deletions (HD) were observed respectively in 55.3\%, 42.1\% and $25.5 \%$ of tumour samples. The most common AMs were on KRAS $(n=156 ; 8.5 \%)$, PIK3CA ( $n=150 ; 8.2 \%)$, CDKN2A HD $(n=174 ; 9.5 \%)$, PTEN HD $(n=49,2.7 \%)$, CCND1 $(n=$ 97; 5.3\%), FGFR1 ( $n=56$; 3.1\%), MDM2 ( $n=53 ; 2.9 \%)$, HER2 $(n=42 ; 2.3 \%)$ and HER1 $(n=41 ; 2.2 \%)$. MTT were recommended in 644 patients. Among them, 101 initiated a recommended MTT. MTT received were $\mathrm{MTOR}$ inhibitors (39\%), anti-angiogenic TKI (21\%), EGFR TKI (9.8\%), and cell cycle inhibitors (6.9\%). Best responses were complete response (CR; $n=2,2.3 \%)$, partial response (PR; $n=13$, $15.1 \%)$, stable disease (SD; $n=29,33.7 \%)$, progressive disease (PD; $n=42,48.8 \%$ ), with a median of progression-free survival (PFS) of 2.8 months (95\% IC: $2.2-3.5$ ). $24 \%$ are alive progression free at 6 months.

Sylvian Dureau and colleagues presented results of the SHIVA trial with survival analysis during ASCO 2017. The SHIVA trial was a proof-of-concept, multicentric randomized phase II trial comparing molecularly targeted therapy among 11 drugs based on tumour molecular profiling versus conventional therapy in patients with any type of cancer that is refractory to standard of care (NCT01771458). The study was conducted at eight aca- demic sites in France. Only patients who had a druggable molecular alteration (DMA) identified on a mandatory tumour sample from a metastatic site using targeted sequencing, $\mathrm{CGH}$ and $\mathrm{IHC}$ were randomized. Cross-over was allowed at disease progression. The trial did not show any difference for its primary endpoint (PFS) [Le Tourneau et al., Lancet Oncol 2015]. At ASCO 2017 the OS of randomized and non-randomized patients was reported. OS was estimated in the 4 following groups: 1) randomized patients, 2) patients for whom a DMA was identified but who were not subsequently randomized because they did not meet the randomization criteria (PS of 0 or 1 , adequate organ function), 3) non-randomized patients because of the absence of DMA, and 4) non-randomized patients because no genomic analyses were performed. Since $70 \%$ of patients randomized to the standard arm eventually crossed over to the targeted therapy arm, all randomized patients were analyzed in group 1. The groups were compared in terms of patient characteristics using Student's t-test and the $\chi^{2}$ test. OS was estimated using the Kaplan-Meier method. Among 741 patients included in SHIVA follow-up data were available for 680 out of the 733 patients. 197, 78, 222 and 183 patients belonged to groups 1 to 4 , respectively. Median OS of the whole cohort was 7.9 months (95\% Cl: 7.0-9.1). As compared to non-randomized patients due to the absence of identified DMA, non-randomized patients with a DMA had a significantly worse prognosis: $H R=2.3(95 \%$ Cl: 1.7-3.0; $p>0.0001)$ whereas randomized patients had a non-significant trend toward a better prognosis: $\mathrm{HR}=0.85$ (95\% Cl: 0.7-1.1; $p=0.18)$. A statistically significant difference in OS was only observed in patients with a DMA who were not randomized. However, the analysis does not show a prognostic value of the DMA on OS. Figure 1 shows SHIVA's primary endpoint - PFS. 


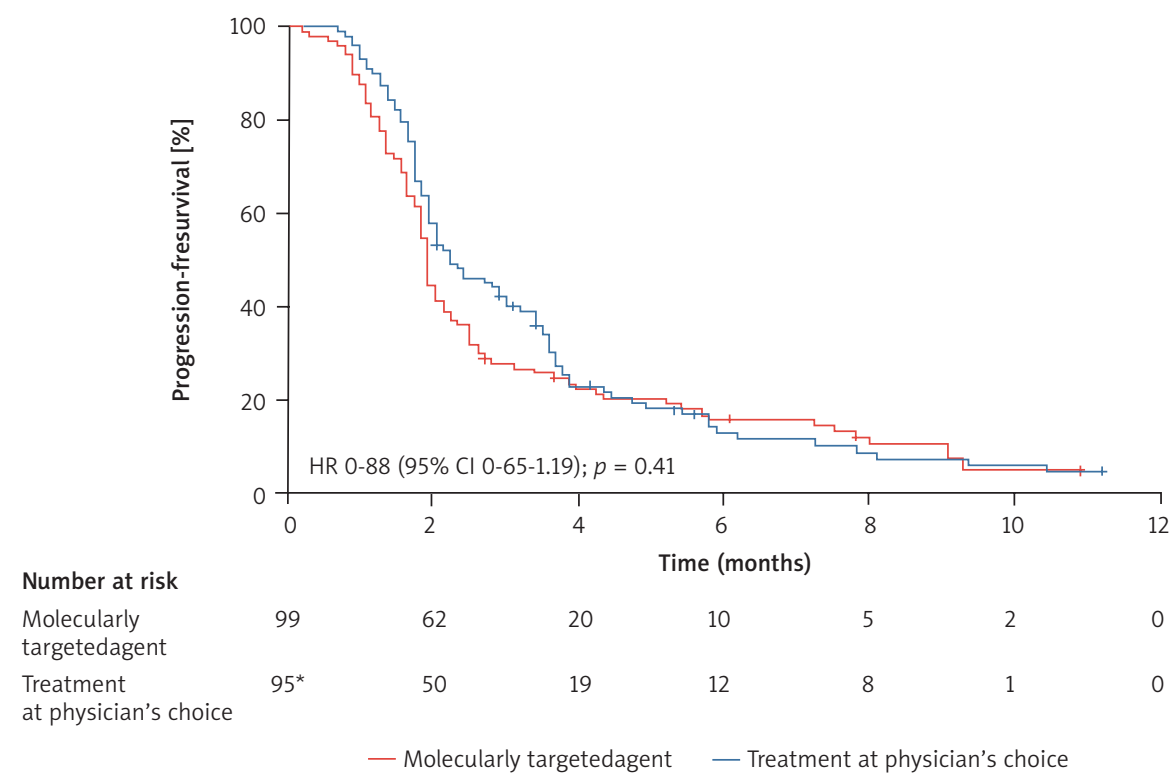

Fig. 1. SHIVA trial results: primary endpoint PFS

Apostolia Tsimberidou and colleagues at ASCO 2017 presented an analysis of treatment results of 637 patients in the IMPACT trial - an MD Anderson precision medicine study. Patients with advanced, refractory cancer who had tumour genomic analyses were treated with matched targeted therapy (MTT) when available. Overall, 1,179 (82.1\%) of 1,436 patients had $\geq 1$ alteration (median age, 59.7 years; men, 41.2\%); 637 had $\geq 1$ actionable aberration and were treated with MTT $(n=390)$ or non-MTT $(n=247)$. Patients treated with MTT had higher rates of CR and PR ( $11 \%$ vs. $5 \% ; p=0.0099$ ), longer failure-free survival (FFS) (3.4 vs. 2.9 months; $p=0.0015$ ), and longer overall survival (OS) (8.4 vs. 7.3 months; $p=0.041$ ) than unmatched patients. Two-month landmark analyses showed that, for MTT patients, FFS for responders vs. non-responders was 7.6 vs. 4.3 months $(p<0.0001)$ and OS was 23.4 vs. 8.5 months ( $p<0.0001$ ); for non-MTT patients (responders vs. non-responders), FFS was 6.6 vs. 4.1 months $(p=0.0005)$ and OS was 15.2 vs. 7.5 months ( $p=0.43$ ). Patients with both
PI3K and MAPK pathway alterations matched to PI3K/Akt/ mTOR axis inhibitors alone showed outcomes comparable to unmatched patients.

The author declares no conflict of interest.

\section{References}

1. Kris MG, Johnson BE, Berry LD, et al. Using multiplexed assays of oncogenic drivers in lung cancers to select targeted drugs. JAMA 2014; 311: 1998-2006.

2. Aisner D, Sholl LM, Berry LD, et al. Effect of expanded genomic testing in lung adenocarcinoma on survival benefit: the Lung Cancer Mutation consortium II experience. J Clin Oncol 2016; 34: Suppl. 11510 [abstract].

3. Le Tourneau C, Delord J-P, Gonçalves A, et al. Molecularly targeted therapy based on tumour molecular profiling versus conventional therapy for advanced cancer (SHIVA): a multicentre, open-label, proof-of-concept, randomised, controlled phase 2 trial. Lancet Oncol 2015; 16: 1324-34.

\section{Box 1. Conclusions from early clinical studies of precision therapies:}

- high numbers of screened cases, high proportions of successfully genetically profiled cases

- low proportion of patients received matched drug

- modest or poor clinical outcomes

- off-label use of targeted drugs based on tumour profiling not supported so far

- clinical trials based on molecular profile earlier in the course of the disease for the future

- combination of targeted therapies needed - but high toxicity issues to be resolved

- deeper understanding of cancer biology and evolution needed

\section{Box 2. Possible factors responsible for poor or modest clinical results of precision therapies:}

Tumours within each cancer patient have been found to be extensively heterogeneous both spatially across distinct regions and temporally in response to treatment. This spatial and temporal heterogeneity poses challenges for prognostic/diagnostic biomarker identification and rational design of optimal drug combinations/clinical trials to minimise reoccurrence. 
4. André F, Bachelot T, Commo F, et al. Comparative genomic hybridisation array and DNA sequencing to direct treatment of metastatic breast cancer: a multicentre, prospective trial (SAFIR01/UNICANCER). Lancet Oncol 2014; 15: 267-74.

5. Meric-Bernstam F, Brusco L, Shaw K, et al. Feasibility of large-scale genomic testing to facilitate enrollment onto genomically matched clinical trials. J Clin Oncol 2015; 33: 2753-62.

6. Bedard PL, Oza AM, Clarke B, et al. Molecular profiling of advanced solid tumors at Princess Margaret Cancer Centre and patient outcomes with genotype-matched clinical trials. Clin Cancer Res 2016; 22: PR03-PRO3 [abstract].

7. Sohal DPS, Rini BI, Khorana AA, et al. Prospective clinical study of precision oncology in solid tumors. J Natl Cancer Inst 2016; 108: pii: djv332.

\section{Address for correspondence}

\section{Sergiusz Nawrocki MD, PhD}

Oncology and Radiotherapy Chair

Medical University of Silesia

Ceglana 35

40-514 Katowice, Poland

e-mail: sergiusz.nawrocki@me.com 